\title{
Maurolico e Pascal: INDUÇÃo MATEMÁTICA E DEMONSTRAÇÃo POR EXEMPLIFICAÇÃo
}

\author{
John A. Fossa \\ Universidade Estadual da Paraíba - UEPB - Brasil
}

(aceito para publicação em outubro de 2021)

\begin{abstract}
Resumo
A Indução Matemática foi aparentemente inventada por Francesco Maurolico e reformulada e reestruturada por Blaise Pascal. Nas suas demonstrações do passo da indução dos seus argumentos, todos os dois recorreram a demonstrações por exemplificação. Nesse sentido, o argumento de Maurolico é satisfatório, enquanto o de Pascal não o é. O método de Pascal, no entanto nos fornece um algoritmo para resolver toda instância particular coberta por sua proposição.
\end{abstract}

Palavras-chave: Técnicas de Demonstração em Matemática, Indução Matemática, Demonstração por Exemplificação, Maurolico, Pascal.

[Maurolico e Pascal:

MathematiCal IndUCTION AND ProOF BY EXEMPLifiCATION]

\begin{abstract}
Mathematical Induction was apparently invented by Francesco Maurolico and reformulated and restructured by Blaise Pascal. In their demonstrations of the induction step of the argument, both had recourse to demonstrations by exemplification. In this regard, Maurolico's argument is satisfactory, but that of Pascal is not. Nevertheless, Pascal's method does provide us with an algorithm for resolving any particular instance covered by his proposition.
\end{abstract}

Keywords: Techniques of Demonstration in Mathematics, Mathematical Induction, Proof by Exemplification, Maurolico, Pascal. 


\section{Introdução}

A Indução Matemática é uma das mais importantes técnicas de demonstração em matemática. Nela, se demonstra que uma proposição é verdadeira para todos os números naturais, ou para todos menos um segmento inicial, por demonstrar $(i)$ que é verdadeira para o primeiro elemento da sequência e (ii) que, se for verdadeira para qualquer elemento da sequência, será verdadeira também para o próximo elemento. A parte $(i)$ é chamada a base da indução. Se usarmos $\mathrm{P}(n)$ para representar "a proposição $\mathrm{P}$ é verdadeira para o número natural $n$ ”, a base da indução é expressa por $\mathrm{P}(1)$ ou, para o caso em que a proposição só vale a partir do número $k, \mathrm{P}(k)$. Em alguns casos é até interessante começar com 0 ou até um inteiro negativo. No entanto, todos os casos alternativos podem ser reduzidos ao caso começando com $\mathrm{P}(1)$, por uma mudança da variável.

Parte (ii) da demonstração é chamada o passo da indução. É frequentemente comparada a uma fila de pedras de dominó, pois, ao derrubar uma pedra, cada uma derruba a próxima. Ela pode ser expressa por $\mathrm{P}(n) \Rightarrow \mathrm{P}(n+1)$, onde a seta indica o condicional material. Na lógica geralmente tomada como subsidiária à matemática, vale o Teorema da Dedução que nos permite desmembrar o antecedente do condicional como uma nova premissa (ou, reciprocamente, eliminar uma premissa por incorporá-la como antecedente do condicional). Fazendo isso, aceitamos $\mathrm{P}(n)$ como premissa, chamada a hipótese da indução. Assim, o passo da indução se reduz à demonstração de $\mathrm{P}(n+1)$ a partir da premissa $\mathrm{P}(n)$.

Parece que a Indução Matemática foi inventada por Francesco Maurolico e reestruturada por Blaise Pascal. No que segue, investigaremos argumentos desses dois matemáticos em relação à Indução Matemática. Descobriremos que todos os dois, devido às limitações simbólicas das suas álgebras, também utilizaram uma outra técnica de demonstração, a demonstração por exemplificação, para fazer o passo da indução. A referida técnica mostra o que acontece numa instância da proposição a ser demonstrada de tal forma que ela possa ser claramente aceita como um template para todos os outros casos. Veremos que Maurolico foi bem sucedido na sua demonstração, enquanto o mesmo não foi o caso para Pascal. Mesmo assim, mostraremos que o procedimento de Pascal nos fornece um algoritmo que pode ser demonstrado válido.

\section{Francesco Maurolico}

O pai de Francesco Maurolico fugiu, ainda jovem, da Grécia para escapar as invasões dos turcos. Fixou-se em Messina, na Sicília, onde se tornou um próspero médico. Francesco nasceu em 1494 e aprendeu a matemática e a astronomia com seu pai e a gramática e a retórica de um padre local. Visto que a sua língua mãe era o grego, teve acesso a obras originais dos grandes escritores gregos, chegando a traduzir (e reorganizar) partes de $O s$ Elementos de Euclides. Foi ordenado padre beneditino em 1521 e pouco depois saiu de Messina para se esquivar de uma praga que assolava a cidade, matando, inclusive, seu pai. A herança que recebeu foi o suficiente para lhe permitir concentrar nas suas atividades científicas por vários anos, embora sempre precisava de patrocínio para a publicação. Em 1540, lutou contra os espanhóis, embora estes permaneceriam na Sicília por muito tempo. Era, por vários anos, diretor da casa da moeda de Messina e um dos responsáveis pelas 
fortificações da cidade. Em 1548 Ignácio de Loyola (1491-1556) fundou, em Messina, o primeiro colégio jesuíta (atualmente a Universidade de Messina). Maurolico era um dos responsáveis pela organização do programa de matemática da nova instituição e foi contratado como professor dela em 1569. Escreveu uma gramática, uma história da Sicília e importantes obras astronómicas, nas quais, contudo, manteve a Terra como o centro do universo e atacava a astronomia copernicana. Na matemática, restaurou obras mal preservadas dos geômetras gregos, incluindo nelas algumas inovações suas, e também escreveu sobre a geometria, a trigonometria e a teoria dos números. Os seus Arithmeticorum libri duo (Dois livros aritméticos) foram escritos em 1557, mas só publicados em 1575, quatro dias após o seu falecimento, nos seus Opuscula mathematica, uma coleção de vários textos matemáticos. ${ }^{1}$

São nos Arithmeticorum libri duo que encontramos a demonstração de Maurolico de que a soma dos primeiros $n$ números ímpares é igual a $n^{2}$. Nas suas próprias palavras, a décima quinta proposição do primeiro livro da referida obra afirma:

"Ex aggregatione imparium numerorum ab vnitate per ordinem successive sumptorum, construuntur quadrati numeri continuati ab vnitate, ipsisq, imparibus collaterales. Nam per antepraemissam, vnitas imprimis cum impari sequente facit quadratum sequentem scilicet, 4 . Et ipse 4. quadratus secundus, cum impari tertio scilicet 5 . facit quadratum tertium, scilicet 9 . Itemque 9. quadratus tertius cum impari quarto scilicet 7 . facit quadratum quartum, scilicet 16 . \& sic deinceps in infinitum, semper $13^{\mathrm{a}}$ repetita propositum demonstratur." (MAVROLYCUS, 1575, p. 7.)

A proposição, portanto, afirma que ao somar os números ímpares em ordem, a partir da unidade, obtemos a sequência dos números quadrados. Assim, a soma dos primeiros $n$ números ímpares é igual a $n^{2}$ (ipsisq, imparibus collaterales). ${ }^{2}$

$\mathrm{Na}$ sua demonstração, ilustra o que acontece nos primeiros casos da sequência, fazendo os cálculos no texto e colocando um diagrama esquemático na margem (ver a Figura 1).

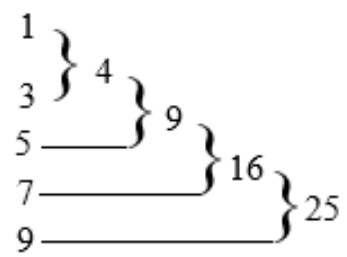

Figura 1. (MAVROLYCUS, 1575, p. 7.)

\footnotetext{
${ }^{1}$ Os detalhes sobre a vida de Maurolico foram retiradas de O’Connor e Robertson (2010).

${ }^{2}$ Na página 3 da sua obra, Maurolico lista os primeiros dez elementos de vários tipos de números, dispondo-os em colunas. Números em colunas diferentes, mas na mesma linha são chamados "colaterais".
}

RBHM, Vol. 21, n 41, pp. 11-24, 2021 
Parece claro que a sua intenção é a de indicar que o procedimento por ele indicado é aplicável a sequência inteira dos números ímpares e que a recorrência infinita do seu raciocínio garante a proposição geral. Desta forma, devemos investigar a sua demonstração mais cuidadosamente para determinar se tenha, como alega W. H. Bussey $(1917)^{3}$, a forma de indução.

\section{A Demonstração de Maurolico}

À primeira vista, a demonstração proposta por Maurolico parece ser uma exposição clássica da Indução Matemática, pois a primeira sentença dela parece afirmar que a soma dos primeiros dois números ímpares, $1+3$, é igual ao quadrado 4 . Isso seria a base da indução. $\mathrm{O}$ restante, então, seria o passo da indução. Nesse restante, no entanto, ele apenas dá mais dois exemplos e finaliza afirmando que o mesmo acontece em todos os outros infinitos casos. Se isso fosse a interpretação correta da sua demonstração, teríamos um exemplo de indução incompleta (ou indução científica), o que não é um argumento dedutivamente válido.

A interpretação dada no parágrafo anterior, contudo, não é plausível devido ao simples fato de que, na demonstração inteira, Maurolico não faz um único cálculo aritmético, nem na primeira sentença (a suposta base da indução), onde poderíamos esperar semelhante cálculo. Em vez disso, ele começa com a unidade, que é simultaneamente o primeiro número ímpar e o primeiro número quadrado, e afirma que a sua soma com o próximo número ímpar (3) é o próximo quadrado (4) devido ao que tem precedido (per antepraemissam). Isto é uma referência à sua décima terceira proposição: "Omnis quadratus cum impari sequente coniunctus, constituit quadratum sequentem" (MAVROLYCUS, 1575, p. 7), ou seja, a soma de qualquer quadrado (implicitamente tomado como um elemento na sequência dos números quadrados) e o próximo ímpar (o que é colateral ${ }^{4}$ com o próximo quadrado) produzirá o próximo quadrado da sequência dos quadrados. Em termos algébricos modernos, isso é simplesmente $n^{2}+2 n+1=(n+1)^{2}$. Maurolico, porém, não tem o recurso da álgebra moderna; mesmo assim, seu raciocínio é claro. Começa com a unidade, considerado como o primeiro quadrado e soma a ela o próximo (o segundo) ímpar (3). Segundo a décima terceira proposição, o resultado será o próximo (o segundo) quadrado (4).

A segunda sentença da demonstração faz mais uma aplicação da mesma décima terceira proposição, pois afirma que o segundo quadrado (4) mais o terceiro ímpar (5) será o terceiro quadrado (9). Na última sentença da demonstração, faz mais uma aplicação da referida proposição, observando que o terceiro quadrado (9) mais o quarto ímpar (7) será o quarto quadrado (16) e ainda afirma que o resultado da proposição a ser demonstrada (a décima quinta) segue por proceder do mesmo modo sucessivamente (sic deinceps).

A demonstração, portanto, é um exemplo de demonstração por exemplificação, em que os exemplos dados servem como um template para mostrar como o procedimento usado

\footnotetext{
${ }^{3}$ No seu artigo, Bussey observa que Moritz Cantor (1829-1920), nas suas Vorlesungen über Geschichte der Mathematik (Vol. 1, 1880) havia indicado Blaise Pascal como o originador da Indução Matemática, mas que ele mesmo havia corregido sua opinião, a favor de Maurolico, num artigo publicado em 1902. Segundo Cantor, ele foi alertado sobre a obra de Maurolico pelo matemático italiano Giovanni Vacca (1872-1953).

${ }^{4}$ Ver a nota 2.
} 
é aplicável em todos os casos. Era bastante usada para efetuar demonstrações gerais em contextos retóricos, na ausência de um simbolismo algébrico mais robusto. Em contraste à indução incompleta, fornece uma demonstração geral porque o template garante o resultado em todos os casos. Podemos exemplificar a diferença com a própria proposição 15 de Maurolico. Se a demonstração tivesse procedido por fazer somas, teríamos algo como o seguinte: " $1+3=4$, o segundo quadrado; $4+5=9$, o terceiro quadrado; $9+7=16$, o quarto quadrado; portanto, ..." A conclusão, contudo, não é válida porque não há qualquer garantia de que as novas somas vão continuar a produzir novos quadrados e só poderíamos saber se isso continue sendo o caso por efetuar as somas e conferir o resultado em cada caso. A demonstração por exemplificação, em contraste, garante o resultado em cada caso pela iteração da décima terceira proposição.

A demonstração de Maurolico é, portanto, completamente geral e, em si (isto é, supondo a validade da décima terceira proposição), válida. Ainda falta, porém, determinar se esse exemplo de uma demonstração por exemplificação é também um exemplo de Indução Matemática.

Para tanto, devemos observar que a referida demonstração não tem a forma moderna tripartida que consiste em uma base, uma hipótese da indução e o passo da indução. Em contraste, consiste em uma cadeia única em que cada elo é ligado ao próximo pela utilização do quadrado produzido, pela décima terceira proposição, em cada elo como o ponto de partida para a produção do próximo quadrado no próximo elo. (Ver a Figura 2.)

$1^{\circ} \square+2^{\circ}$ impar gera $\underset{\text { prop. } 13}{2 \circ}$

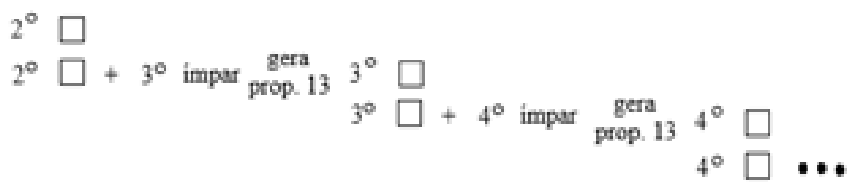

Figura 2. A forma da demonstração de Maurolino.

Ao cogitar essa cadeia infinita em que cada elo é gerado a partir do elo anterior, Maurolico certamente estava de posse da ideia fundamental da Indução Matemática, embora a expressava de forma elíptica na sua exposição retórica.

\section{Blaise Pascal}

Em 1623, quase cinquenta anos após o falecimento de Maurolico, Blaise Pascal nasceu na cidade de Clermont, no sul da França. Eventualmente, seu pai, Étienne, mudou para Paris para providenciar melhores oportunidades educacionais para seus filhos. Lá, ele foi um membro ativo das reuniões científicas promovidas pelo frade Marin Mersenne (1588-1648) e, visto que Blaise mostrou habilidades extraordinárias ainda muito jovem, também levava 
seu filho a participar nessas reuniões. Nelas, o jovem Blaise conheceu as teorias e descobertas de alguns dos mais importantes cientistas da época e ainda travou uma forte amizade com Pierre de Fermat (1601-1665). Pascal adotava o então emergente metodologia científica e a aplicou à construção de uma máquina de calcular, a um projeto de iluminar as ruas de Paris durante a noite, ao estabelecimento de um sistema de transporte público e ao uso do barômetro de Evangelista Torrecelli (1608-1647) para provar a existência do vácuo (o que lhe incorreu a inimizade de René Descartes) e para criar a ciência da hidrostática. Em 1654, Pascal teve uma experiência mística que o levou a abandonar as questões científicas e dedicar todos os seus esforços à exploração de problemas religiosos. Dois das suas obras mais aclamadas, as Lettres provinciales e as Pensées foram escritas nesse período. Pascal veio a falecer precocemente em $1662 .{ }^{5}$

Na matemática, trabalhou na geometria projetiva e, junto com Fermat, criou a teoria da probabilidade. Também investigou o triângulo numérico que atualmente leva o seu nome. $\mathrm{O}$ triângulo era conhecido antes de Pascal, mas foi ele que fez um estudo sistemático dele e mostrou como ele poderia ser aplicado a diversas áreas da matemática. É nessa obra, o Traité du Triangle Aritmetique (1665) por Pascal. Para melhor apresentar o procedimento de Pascal, fazemos antes uma rápida revisão do triângulo aritmético como apresentado por Pascal.

\section{O Triângulo de Pascal}

O triângulo consiste em um meio quadrado (infinito), dividido em "células" (cellules), cada umas das quais contendo um número, conforme a Figura 3. As linhas de células são denominadas de "fileiras paralelas" (rangs paralleles), enquanto as respectivas colunas são chamadas de "fileiras perpendiculares" (rangs perpendiculaires). Os números acima do meio quadrado, bem como os à esquerda dele, não fazem parte do próprio triângulo, mas servem para enumerar as fileiras. Pascal os chama de "expoentes" (exposans). Finalmente, as linhas diagonais de células que se encontram entre dois expoentes do mesmo valor são chamadas de "bases" (bases). Assim, cada base determina um segmento inicial do triângulo.

O Traité é, basicamente, uma obra retórica e, especificamente, não usa tais símbolos como $a_{i j}$ para referir as células. Assim, usa expressões retóricas para identificar as células individuais; em alguns casos, coloca uma letra latina ou uma letra grega numa célula para a identificar. O conteúdo de cada célula é determinado recursivamente. O número a ser colocado na célula $\mathrm{G}$ (ver a Figura 3) é arbitrário, mas, nos seus escritos sobre o triângulo, ele sempre coloca a unidade nessa célula. As outras células serão preenchidas segundo a seguinte regra: o número de cada célula é a soma do número da célula que a precede na mesma fileira paralela e o número da célula que a precede na mesma fileira perpendicular.

\footnotetext{
${ }^{5}$ Para mais detalhes sobre a vida e obra de Pascal, ver, por exemplo, Fossa (2013).

${ }^{6}$ Embora publicado em 1665, o Traité foi escrito em 1653.
} 


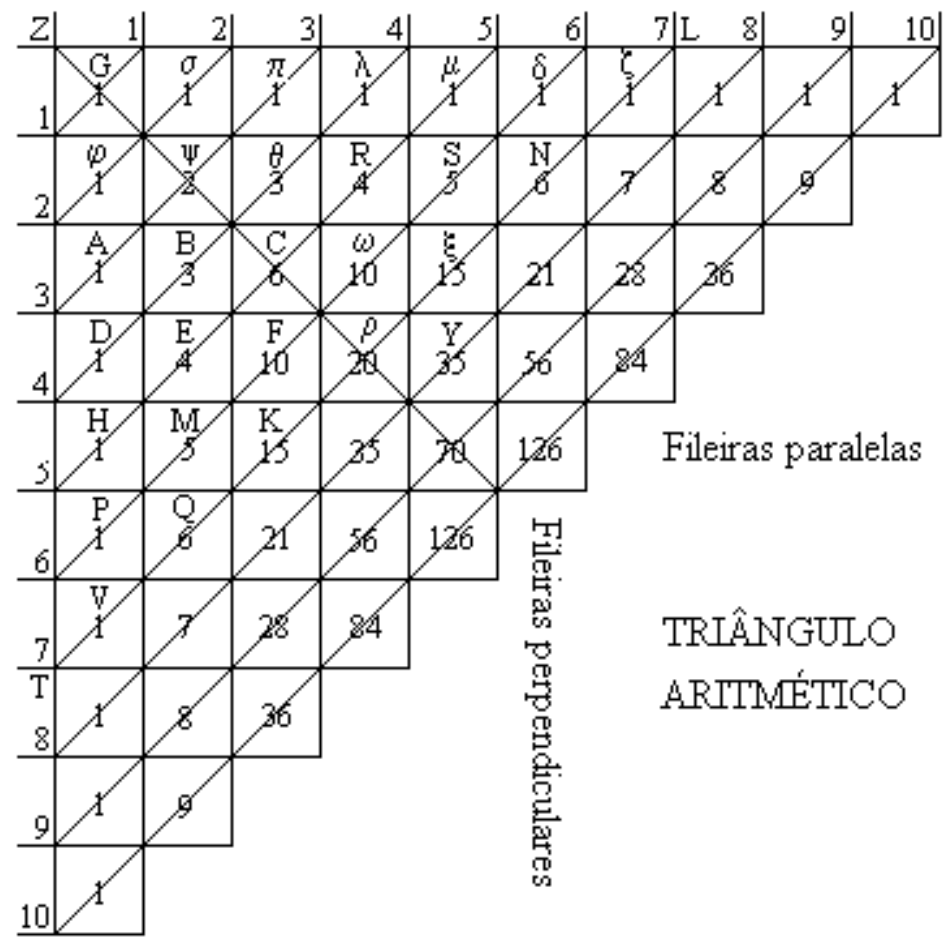

Figura 3. O Triângulo de Pascal. Fonte: PASCAL (2013, p. 54).

Da lei de formação do triângulo, Pascal tira várias consequências. É a décima segunda delas que será do nosso interesse. Afirma que:

"Em tout Triangle Arithmetique, deux cellules contigues estant dans vne mesme dase, la superieure est à l'inferieure, comme la multitude des cellules depuis la superieure iusques au haut de la base, à la multitude de celles, depuis l'inferieure iusques en bas inclusiuement." (PASCAL, 1665, p. 7.)

Assim, segundo a proposição, a razão entre os números em células contíguas numa mesma base será igual à razão entre a quantidade de células das partes da base determinadas pelas duas células originais. As células $\mathrm{C}$ e $\mathrm{E}$, por exemplo, particionam a quinta base em duas partes, uma contendo as três células $\mathrm{C}, \mathrm{R}, \mu$ e a outra contendo as duas células $\mathrm{E}, \mathrm{H}$. Assim, a base é particionada na razão 3:2. Os números contidos nas células C (6) e E (4) têm a mesma razão. Observamos que a proporção é formulada na proposição com a razão superior para inferior, mas, no exemplo e da demonstração, ele a formula, às vezes, com a razão inferior para superior. Isso, como deve ser evidente, é completamente imaterial. 


\section{A Demonstração de Pascal}

A seguir, Pascal reconhece que a demonstração acarretaria um número infinito de casos particulares, mas afirma que poderá ser feita, de forma geral, utilizando dois lemas. Nas suas próprias palavras:

Quoy que cette propositition ait vne infinité de cas, i'en donneray vne demonstration bien courte, en supposant 2 lemme.

Le 1. que est euident de soy-mesme, que cette proportion se rencontre dans la second base; car il est bien visible que $\varphi$ est à $\sigma$ comme 1 , à 1 .

Le 2. que si cette proportion se trouue dans vne base quelconque, elle se trouuera necessairement dans la base suiuante.

D'où il se voit, qu'elle est necessairement dans toutes les bases: car, elle est dans la seconde base, par le premier lemme, donc par le second elle est dans la troisiesme dase, donc dans la quatriesme, \& à infiny. (PASCAL, 1665, p. 7.)

Disto, fica claro que Pascal era consciente da estrutura da sua demonstração e que essa estrutura é formulada de maneira equivalente à formulação moderna. Em especial, o primeiro lema corresponde à base da indução, enquanto o segundo corresponde ao passo da indução. A única diferença entre a formulação moderna usual tripartida e a de Pascal é estética, pois temos

$$
\begin{array}{ll}
\text { formulação moderna } & \text { formulação de Pascal } \\
\begin{array}{ll}
\text { 1. } \mathrm{P}(1) & 1 . \mathrm{P}(1) \\
\text { 2. } \mathrm{P}(n) \text { (hipótese) } & \text { 2. } \mathrm{P}(n) \Rightarrow \mathrm{P}(n+1) \\
\text { 3.P }(n+1) &
\end{array}
\end{array}
$$

e, para a lógica subsidiária à matemática, como já vimos, o desmembramento do antecedente do condicional como uma premissa é absolutamente equivalente ao condicional. De fato, muitos textos modernos usam a formulação de Pascal.

No último parágrafo do trecho citado, Pascal explica a lógica por trás do argumento. Podemos explanar seu raciocínio esquematicamente da seguinte forma:

$$
\begin{aligned}
& \mathrm{P}(1) \\
& \mathrm{P}(1) \Rightarrow \mathrm{P}(2) \\
& \mathrm{P}(2) \\
& \mathrm{P}(2) \Rightarrow \mathrm{P}(3) \\
& \mathrm{P}(3) \\
& \mathrm{P}(3) \Rightarrow \mathrm{P}(4)
\end{aligned}
$$


Assim, Pascal aponta para uma cadeia infinita em que a conclusão de cada elo vira premissa no elo seguinte. Isto se assemelha muito à cadeia infinita proposta na demonstração de Maurolico, o que nos leva a pensar que Pascal conhecia o trabalho de Maurolico e, de fato, Bussey (1917) cita uma carta de Pascal em que ele faz referência à esse tipo de demonstração na obra de Maurolico. Dessa forma, na ausência de evidência de que Indução Matemática fosse usada antes, Maurolico deverá ser considerado como o originador desse tipo de demonstração. Mesmo assim, parece que Pascal era mais consciente da Indução Matemática como um poderoso tipo de argumentação matemática e, certamente, deu a ela uma formulação mais geral e, portanto, mais aplicável a diversas situações matemáticas. Observamos que Pascal era também ciente do fato de que a base nem sempre reza sobre a unidade, mas sobre o primeiro elo da cadeia. No caso sob consideração, a base da indução afirma que a sua proposição 12 é verdadeira para a segunda base. De fato, nem é aplicável à primeira base, pois esse consiste em uma única célula e, portanto, não contém duas células contíguas.

\section{A Demonstração do Segundo Lema}

Como é frequentemente o caso em demonstrações por indução, a demonstração do primeiro lema de Pascal é evidente por inspeção. Assim, voltamos a nossa atenção para a sua demonstração do segundo lema. Será necessário demonstrar, então, que sempre que a proposição 12 for verdadeira para uma dada base, será verdadeira também para a base seguinte. Ele faz isso da seguinte maneira:

Il faut donc seulment demonstrer le second lemme, en cette sorte. $\mathrm{Si}$ cette proportion se rencontre en vne base quelconque, comme en la quatriesme $\mathrm{D} \lambda$, c'est à dire, si $\mathrm{D}$ est à $\mathrm{B}$ comme 1 à 3 . Et $\mathrm{B}$, à $\theta$ comme 2 à 2 . Et $\theta$ à $\lambda$ comme 3 à 1 . \&c.

Ie dis, que la mesme proportion se trouuera dans la base suiuante, $\mathrm{M} \mu, \&$ que par exemple $\mathrm{E}$ est à $\mathrm{C}$ comme 2 à 3 .

Car $\mathrm{D}$ est à $\mathrm{B}$ comme 1 à 3 . par l'hypothese.

Donc $\mathrm{D}+\mathrm{B}$ est à $\mathrm{B}$ comme $1+3$ à 3

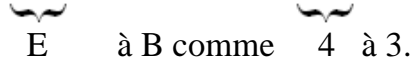

De mesme, $\mathrm{B}$ est à $\theta$ comme 2 à 2 par l'hypothese.

Donc, $\mathrm{B}+\theta$ à $\mathrm{B}$ comme, $2+2$ à 2 .

$\widetilde{C}$ à $\mathrm{B}$ comme 4 à 2

Mais $\mathrm{B}$ à $\mathrm{E}$ comme 3 à 4 comme il est monstré.

Donc par la proportion troublée, C est à E comme 3 à 2.

Ce qu'il falloit demonstrer.

Isto é, Pascal precisa mostrar que, se a proposição valer para todo par de células contíguas em uma base qualquer (quelconque), então também valerá para todo par de células contíguas na próxima base, pois a indução é sobre as bases dos segmentos iniciais do triângulo 
aritmético. Para tanto, ele faz uma demonstração por exemplificação contendo dois particularidades, a saber, $(i)$ escolha a quarta base para representar a hipótese da indução e (ii) escolha o par de células contíguas E, C para representar todos os pares de células contíguas da quinta base.

Referente a $(i)$, devemos observar que, apesar de escolher uma base particular, a quarta, para representar a hipótese da indução, Pascal tem o cuidado a não calcular as proporções nela contidas, mas supõe que as proporções são de acordo com a proposição a ser demonstrada. Isto é, ele não afirma, por exemplo, que $\mathrm{D}$ é para $\mathrm{B}$ como 1 é para 3 porque $\mathrm{D}$ $=1$ e $\mathrm{B}=3$, ou que $\mathrm{B}$ é para $\theta$ como 2 é para 2 porque $\mathrm{B}=3$ e $\theta=3$. Muito pelo contrário, ele apenas diz que supõe que $\mathrm{D}$ é para $\mathrm{B}$ como 1 é para 3 (si $D$ est à $B$ comme 1 à 3 ), etc. Isto é apropriado porque se trata aqui da hipótese da indução.

Referente a (ii), Pascal precisa mostrar que a referida proporção se obtém para todos os pares de células contíguas da quinta base e propõe fazer isto por ilustrar o procedimento geral com o par E, C. Como já vimos, esse par particiona a quinta base em duas partes C, R, $\mu$ e E, H. Assim, é necessário demonstrar que $\mathrm{C}$ é para $\mathrm{E}$ como 3 é para $2 .^{7}$ Ainda mais, deve demostrar que essa proporção se obtém porque a proposição 12 vale na base anterior, a quarta. Se tivesse dito que $C: E:: 3: 2$ porque $C=6$ e $E=4$, ou seja por calcular as razões envolvidas, teria uma demonstração por indução incompleta, um procedimento inválido para a matemática.

Agora Pascal observa, implicitamente ${ }^{8}$, que D, B é um par de células contíguas da quarta base e elas particionam a quarta base nas duas partes $\mathrm{D}$ e $\mathrm{B}, \theta, \lambda$. Assim, pela hipótese da indução temos, expressando, por comodidade, a proporção por uma equação,

$$
\frac{D}{B}=\frac{1}{3}
$$

Disto conclui, por proposição V.18 de Os Elementos de Euclides, que

$$
\frac{D+B}{B}=\frac{1+3}{3} .
$$

Mas, pela lei da formação do triângulo, $\mathrm{D}+\mathrm{B}=\mathrm{E}$. Assim, temos

$$
\frac{E}{B}=\frac{4}{3}
$$

De novo, o par de células contíguas $\mathrm{B}, \theta$ particiona a quarta base nas partes $\mathrm{B}, \mathrm{D}$ e $\theta, \lambda$. Logo, pela hipótese da indução,

$$
\frac{B}{\theta}=\frac{2}{2}
$$

\footnotetext{
${ }^{7}$ Aqui seguimos Pascal em usar o nome das células para representar também os números neles contidos.

${ }^{8}$ Vários detalhes da demonstração são deixados implícitos por Pascal. A nossa exegese fará explícitos alguns deles.
} 
Mas, isto é equivalente a $\frac{\theta}{B}=\frac{2}{2}$. Assim, pela mesma proposição V.18, obtemos

$$
\frac{B+\theta}{B}=\frac{2+2}{2}
$$

Visto que $\mathrm{B}+\theta=\mathrm{C}$, pela lei de formação do triângulo, temos

$$
\frac{C}{B}=\frac{4}{2}
$$

Assim, temos

$$
\left\{\begin{array}{l}
\frac{C}{B}=\frac{4}{2} \\
\frac{B}{E}=\frac{3}{4}
\end{array} .\right.
$$

Logo, pela proposição V.23 de Os Elementos de Euclides (la proportion troublée), obtemos o resultado desejado:

$$
\frac{C}{E}=\frac{3}{2}
$$

Fica evidente, portanto, que Pascal concluiu que $\frac{C}{E}=\frac{3}{2}$ a partir da hipótese da indução. Como uma demonstração por exemplificação, no entanto, seu procedimento não é bem sucedido porque não fica evidente se o mesmo poderá ser aplicado a outras bases do triângulo. Isso acontece porque os cálculos numéricos utilizados no procedimento não são apresentados de tal forma a sustentar a generalização necessária.

Desta forma, julgamos que o procedimento de Pascal para demonstrar o segundo lema, referente à sua proposição 12, não é uma demonstração por exemplificação legítima, embora ele claramente intencionou fazer esse tipo de demonstração. Mesmo assim, o procedimento fornecido por Pascal sempre funciona. Isso poderá parecer um tanto paradoxal, pois se o procedimento sempre funciona, porque não é uma demonstração legítima? Para entender isso, devemos lembrar que o propósito de uma demonstração é o de averiguar que a conclusão é uma consequência válida das premissas. Assim, é possível que um procedimento sempre gera resultados válidos sem mostrar a sua validade. Parece-nos que é esse o caso da demonstração proposta por Pascal para o referido segundo lema. Desta forma, é defeituosa na medida que carece de maiores explicações.

\section{A Validade do Procedimento de Pascal}

Nos últimos dois parágrafos, alegamos que o procedimento que Pascal usou para tentar demonstrar o segundo lema sempre gera resultados corretos, embora esse fato não seja evidente. Assim, será procedente mostrar que o referido procedimento de fato fornece 
resultados corretos. Para tanto, usaremos $a_{\text {in }}$ para representar o $i$-ésimo ${ }^{9}$ elemento da $n$-ésima base. Desta forma, $a_{24}$, por exemplo, é o segundo elemento da quarta base, ou seja, o elemento na célula B do diagonal contendo as células D, B, $\theta, \lambda$ (ver a Figura 3). Podemos observar que nessa notação, $a_{\text {in }}$ aparece na $i$-ésima coluna.

Dado qualquer base $n$, dois elementos contíguos serão representados por $a_{\text {in }}, a_{i+1 n}$. Visto que a $n$-ésima base tem $n$ elementos, $a_{\text {in }}, a_{i+1 n}$ particiona a $n$-ésima base nas partes $a_{1 n}, a_{2 n}, \ldots, a_{\text {in }}$ e $a_{i+1 n}, a_{i+2 n}, \ldots, a_{n n}$. Em consequência, a primeira parte tem $i$ elementos, enquanto a segunda parte tem $n-i$ elementos.

Lembramos que o segundo lema é o passo da indução de uma demonstração por Indução Matemática. Assim, supomos que a proposição 12 (que dois elementos contíguos de uma mesma base tem a mesma razão que a quantidade de elementos nas suas respectivas partes) se obtém na $n$-ésima base. Precisamos mostrar que a proposição também se obtém na n+1-ésima base. Ver a Figura 4.

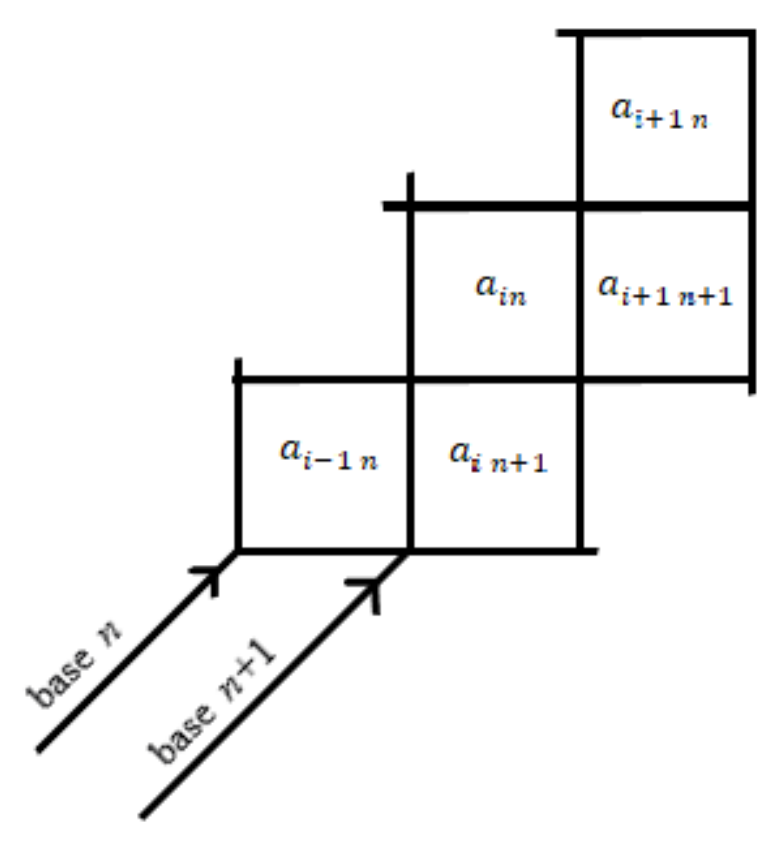

Figura 4. Recorte do Triângulo de Pascal.

\footnotetext{
${ }^{9}$ Contamos de baixo para cima ao longo da diagonal.
} 
Seguiremos, precisamente, o procedimento de Pascal, usando nossa notação generalizada. Pela hipótese da indução, temos

$$
\frac{a_{i-1 n}}{a_{\mathrm{in}}}=\frac{i-1}{n-(i-1)}
$$

Assim, por Euclides V.18 e a lei de formação do triângulo, obtemos

$$
\frac{a_{i n+1}}{a_{\text {in }}}=\frac{a_{i-1 n}+a_{\text {in }}}{a_{\text {in }}}=\frac{i-1+n-(i-1)}{n-(i-1)}=\frac{n}{n-(i-1)} .
$$

De novo, pela hipótese da indução,

$$
\frac{a_{i n}}{a_{i+1 n}}=\frac{i}{n-i}
$$

Assim, por Euclides V.18 (invertendo a proporção) e a lei de formação do triângulo, obtemos

$$
\frac{a_{i+1 n+1}}{a_{\mathrm{in}}}=\frac{a_{i+1 n}+a_{\mathrm{in}}}{a_{\mathrm{in}}}=\frac{(n-i)+i}{i}=\frac{n}{i}
$$

Isto é, temos (invertendo a antepenúltima proporção destacada)

$$
\left\{\begin{array}{c}
\frac{a_{i+1 n+1}}{a_{i n}}=\frac{n}{i} \\
\frac{a_{i n}}{a_{i n+1}}=\frac{n-(i-1)}{n}
\end{array} .\right.
$$

Logo, por Euclides V.23 (la proportion troublée), obtemos:

$$
\frac{a_{i n+1}}{a_{i+1 n+1}}=\frac{i}{n-(i-1)}=\frac{i}{(n+1)-i}
$$

Mas, visto que a parte da partição contendo $a_{i n+1}$ contém $i$ elementos, enquanto a parte contendo $a_{i+1 n+1}$ contém $(n+1)-i$ elementos, a proposição 12 vale para a base $n+1$. Assim sendo, o procedimento de Pascal sempre fornece resultados corretos.

\section{Conclusão}

É frequentemente o caso que inovações matemáticas não nascem completamente aperfeiçoadas na sua primeira aparência na história, mas que sofrem reformulações e melhoramentos nas mãos de investigadores subsequentes. É precisamente isso que tem acontecido com a técnica de demonstração por Indução Matemática. O originador parece ter sido Francesco Maurolico, pois ele claramente usou argumentos consistindo em cadeias infinitas em que cada elo é consequente do elo precedente, criando assim um "efeito dominó". No contexto do seu uso do novo método, porém, Maurolico não fez uma reflexão maior sobre 
a estrutura e validade da sua inovação. Uma reflexão desse tipo foi feita por Blaise Pascal, pois ele aparentemente buscou a ideia básica da Indução Matemática nos escritos de Maurolico e, então, a reformulou de tal forma que ficasse melhor estruturada e, consequentemente, mais geral e mais aplicável a diversas partes da matemática.

Ambos Maurolico e Pascal usaram uma álgebra retórica para expor os seus resultados e isso acarretou no uso de demonstrações por exemplificação nas suas respectivas demonstrações. Para tanto, o template apresentado por Maurolico é evidentemente aplicável ao número infinito de casos cobertos pela sua proposição. Em contraste, o template apresentado por Pascal não alcança um nível semelhante de clareza sobre a aplicabilidade do mesmo. Assim, o procedimento de Pascal não é uma demonstração bem sucedida. É, no entanto, um algoritmo que sempre retorna resultados corretos, como é facilmente demonstrado usando métodos algébricos modernos.

\section{Referências}

BUSSEY, W. H. 1917. The Origin of Mathematical Induction. American Mathematical Monthly. V. XXIV, n. 5. pp. 199-207.

MAUROLYCUS, Franciscus. 1575. Arithmeticorum libri duo. Veneza. Disponível em: <books.google. com.br>. Acesso em 16 de 10 de 2020.

O’CONNOR, J. J, e ROBERTSON, E. F. 2010. Francesco Maurolico. Disponível em: <https://mathshistory.st-andrews.ac.uk/Biographies/Maurolico/>. Acesso em 16/10/2020.

PASCAL, Blaise. 1665. Traité dv Triangle Arithmetique. Paris: Gvillavme Desprez.

John A. Fossa

Universidade Estadual da Paraíba - UEPB - Brasil

E-mail: jfossa03@gmail.com 